\title{
Erratum: Molecular Hydrogen Outside the Near-infrared
}

\author{
Michael Burton \\ Anglo-Australian Observatory and School of Physics, \\ University of New South Wales, PO Box 1 , \\ Kensington, NSW 2033
}

The following corrections should be noted to the above paper which appeared in Proc. Astron. Soc. Aust., Volume 10, Number 4, pages 322-24 (1993).

Abstract: This paper discusses the observation of molecular hydrogen line emission outside near infrared wavelengths, and in particular the opportunities afforded to molecular astrophysics by studies in the mid-infrared and far-red.

Section 1, paragraph 2, line $18: \ldots$ the far-red optical CCD regime, but are considerably weaker in intensity. This paper reports recent developments which extend $\mathrm{H} 2$ observations outside the nearinfrared, to the mid-infrared and far-red regimes.

Section 1, paragraph 5, line 1: There are two particular regimes which this paper concentrates upon, the mid-infrared and the far-red....

Section 1, paragraph 5, line 8: Suitable lines are emitted in the far-red,....

Section 3, Title: Far-red Molecular Hydrogen Line Emission.

Section 3, paragraph 1, line 6: Recently Burton et al. (1992a) observed, for the first time, line emission in the far-red spectral region,....

Section 3, paragraph 1, line $12: \ldots$ which includes the near-infrared data of Howe et al. and the far-red data of Burton et al.

Section 4, paragraph 1, line 10: Far-red lines extend the level population distribution probed.... 